depression than that inferred from examination of prescription drug claims data. The PE literature would be improved in reliability and transparency if each published study described (a) the methods used to reduce selection bias; (b) treatment of the missing data; (c) the key assumptions and data sources; (d) the disaggregated costs and outcomes, comparing the new therapy with the existing one; and (e) where relevant, the costs, consequences, and incremental cost-effectiveness ratio by patient subgroup..$^{25}$

\section{- Prescription Drug Expenditures, Financial Burden, and Health Plan Satisfaction Among Medicare Beneficiaries}

Researchers have for years been studying the milieu of factors associated with the large variation in prescription drug utilization among Medicare beneficiaries. There is potential for greater financial burden imposed by prescription drugs among the elderly who on average use 3 times the number of prescriptions per month compared with persons younger than 65 years. ${ }^{26}$ But the distribution of drug expenditures and financial burden is not uniform. Seventeen percent of Medicare beneficiaries had no $(\$ 0)$ spending on prescription drugs in calendar year 2001, while spending of $\$ 1,000$ or more was found among $28 \%$ of Medicare beneficiaries and accounted for $76 \%$ of total expenditures for prescription drugs for this population. ${ }^{27}$ According to data obtained from the Medicare Current Beneficiary Survey, $76 \%$ of all Medicare beneficiaries had some drug coverage at some point in 1999, up from 73\% in 1997 and 1998 and considerably higher than the $57 \%$ reported in $1992 .{ }^{28}$ About one third of the supplemental prescription drug coverage among Medicare beneficiaries was employer-sponsored and 17\% (12.8\% of all Medicare beneficiaries) had drug coverage through a Medicare+Choice plan). But, Medicare+Choice plans became unavailable to about 2.5 million Medicare beneficiaries between 1998 and 2002, ${ }^{29}$ and employer-sponsored prescription drug benefits for Medicare beneficiaries aged 65 to 69 years declined from $40 \%$ in 1996 to $35 \%$ in $2000 .{ }^{30}$

For the 5-year period through 2003, Medicare+Choice plans with any prescription drug coverage declined from $73.4 \%$ in 1999 to $66.1 \%$ in 2003, and the percentage with an annual maximum drug benefit maximum increased from $23.3 \%$ to $70.0 \%{ }^{31}$ When weighted by enrollment, $83.9 \%$ of Medicare+ Choice members had a prescription drug benefit in 1999, dropping to $68.9 \%$ in 2003. In 1999, only $10.6 \%$ of Medicare+ Choice members had a limited drug plan benefit with a $\$ 500$ annual maximum, but by 2003, 53.4\% of Medicare+Choice members had an annual drug benefit maximum of $\$ 500$. In the Commonwealth Fund data, a relatively small portion of Medicare+Choice plans applied the annual benefit maximum through a quarterly cap, affecting 12.2\% of members in 1999 and $8.8 \%$ of members in 2003, but these data differ from other data sources that show that among HMOs that imposed drug benefit maximums (83\% of plans affecting more than two thirds of membership) in 2001, 42\% imposed an annual cap (average
$\$ 1,160$ ), $47 \%$ had quarterly caps (average \$351), and 11\% imposed monthly caps (average \$80). ${ }^{32}$

Cost sharing in the form of copayments per prescription has also increased. The average generic copay was $\$ 10$ or less for $84 \%$ of members in 1999 versus $72 \%$ in 2003 . The brand copay was $\$ 20$ or more for $14 \%$ of members in 1999 , rising to $74 \%$ of members in 2003. An emerging trend was found in coverage of generic drugs only, affecting $11 \%$ of Medicare+Choice members in 1999 , rising to $41 \%$ in 2003; in 2002, $90 \%$ of members with a generic-only benefit had no annual benefit maximum. Among basic Medicare+Choice plans, $60 \%$ covered only generic drugs in 2003. Copayments for primary care and specialist office visits also increased, and $82 \%$ of members were subject to some type of cost sharing for inpatient hospital admissions in 2003.

Commercial health plans are reluctant to drop coverage of prescription drugs for Medicare beneficiaries for a multitude of reasons, including the perceived value of these benefits among members and the apparent favorable influence that drug coverage has on member satisfaction. A survey in 2001 of 3,457 adults aged 19 years or older, including Medicare-eligible beneficiaries, found that Medicare beneficiaries who had prescription drug coverage were 50\% more likely than those without it to rate their insurance as excellent and about one half as likely to report medical bill problems. ${ }^{33}$ The percentage of Medicare enrollees aged 65 years or older who rated their insurance as excellent was 36\% among members enrolled in plans with prescription coverage compared with $22 \%$ for members in health plans without prescription drug coverage. The percentage of Medicare enrollees aged 65 years or older who reported any medical bill problem was $15 \%$ for plans with prescription drug coverage versus 29\% for Medicare members in health plans without prescription drug coverage.

In a previous issue of the Journal, McKercher, Taylor, Lee, Chao, and Kumar found that prescription drugs in elderly families accounted for approximately twice the proportion of total out-of-pocket medical care burden compared with nonelderly families, $45.6 \%$ and $23.7 \%$, respectively. The higher proportion of total medical care burden and total economic burden attributable to prescription drugs in the elderly was traced to larger prescription quantities, price, and utilization, but not more expensive drugs. In this issue of the Journal, Schommer, Mott, Hansen, and Cline found the proportion of senior citizens without any prescription insurance coverage did not change significantly-from $32 \%$ in 1998 to $29 \%$ in $2001 .{ }^{34}$ However, the proportion of respondents with prescription drug coverage that had to share costs of prescriptions through copayments and coinsurance rose significantly-from 69\% in 1998 to $89 \%$ in 2001.

A key question that begs for useful answers is what behaviors result when persons with prescription drug needs encounter financial barriers to access. Schommer, Mott, Hansen, and Cline found that the proportion of senior citizens who used mail-order pharmacies increased from $17 \%$ in the 1998 survey to $27 \%$ in the 2001 survey. A Harris Interactive 
poll of 3,465 Californians aged 18 years or older with chronic illness, conducted in November and December 2002, found that nearly one half (46\%) reported doing nothing when faced with an increase in drug cost sharing. ${ }^{35}$ (The specific survey question stated that the survey respondent had prescription drug coverage and the amount paid to fill a prescription increased in the past year.) More than one third of respondents (36\%) reported that they asked their physician or pharmacist for a generic prescription drug, $21 \%$ asked the physician to prescribe a less-expensive alternative drug, $16 \%$ did not get the prescription filled, and $18 \%$ used mail-order service to fill the prescription.

In a previous issue of the Journal, Cox and Henderson found that Medicare+Choice members with an annual drug benefit maximum relied in part on prescription drug samples to mitigate the financial burden of prescription drug needs. ${ }^{36}$ This practice is likely to be self-defeating in reducing the financial burden since (a) higher-cost drugs are more heavily sampled and (b) the availability of drug samples may affect physician prescribing practices by reducing the immediate pressure to find lower-cost therapeutic alternatives. ${ }^{37}$ We await the results of research in which investigators ask the important questions regarding the nature and usefulness of interactions with physicians in offering recommendations for generic drugs and other lower-cost therapeutic alternatives to help reduce out-of-pocket expenditures for the elderly.

\section{Hypertension, Prescription Drug Copayments, and Drug Therapy Adherence}

In this issue of the Journal, Wogen, Kreilick, Livornese, Yokoyama, and Frech found that drug therapy adherence differs by type of drug. ${ }^{38}$ In this study, a higher rate of adherence to therapy was found for valsartan compared with lisinopril or amlodipine. Several factors can affect adherence to drug therapy aside from the safety and effectiveness of the drug. A factor not addressed in the extant study, but a curious one is the relationship of member cost share to drug therapy adherence. Some drug industry analysts attributed the small increase in U.S. sales of the block-buster drug atorvastatin (Lipitor, for hypercholesterolemia) in 2003 Q1 and declines in sales of category leaders sertraline (Zoloft, for depression), and amlodipine (Norvasc, for blood pressure) during this period to rising copayments in drug benefit plans. ${ }^{39}$ One wonders how formulary status and copay design (e.g., relative copayments) might have influenced the results observed by Wogen, Kreilick, Livornese, Yokoyama, and Frech, particularly since one of their study drugs was amlodipine. Neither formulary status nor copay design was reported, and one of the study medications, lisinopril, became available in generic form during the study period, but not until the 35 th month of the 36-month study period. It is reasonable to speculate that adherence with generic lisinopril, with its lower copayment, would have been more resilient over time compared with brand drugs amlodipine and valsartan, both with higher (brand drug) copayments.

There are also data to suggest that pharmacy provider type, mail-service versus community pharmacy, another feature of drug benefit design, may influence drug therapy adherence. ${ }^{40}$ This variable, certainly relevant in the database employed by Wogen, Kreilick, Livornese, Yokoyama, and Frech (a pharmacy benefit manager database with a high penetration of mailservice prescriptions), was not measured in the current study. Future studies of drug therapy adherence derived from drug claims data might also include a measure of the average member cost share per day of therapy, in dollar amount and percentage, for the target drugs in the study. This variable has become increasingly important with the proliferation of multi-tier copay drug plans and sometimes widely disparate copayments for generic versus preferred brand versus nonformulary drugs. Managed care pharmacists also need to know if and how mailservice pharmacy is a factor in drug therapy adherence, through the simple convenience of a 90-day supply or home delivery versus store purchase or a lower cost-share requirement. ${ }^{41}$

There are also the important subjects of midpoint and endpoint clinical outcomes, none of which were measured in the current study. A study of blood pressure control in a New York health maintenance organization (HMO) in 1998 found that only $35 \%$ of patients reached target blood pressure $(<140 \mathrm{~mm}$ $\mathrm{Hg}$ systolic and $90 \mathrm{~mm}$ diastolic for the general population and $<130 / 85 \mathrm{~mm} \mathrm{Hg}$ in diabetics and patients with renal insufficiency). ${ }^{42}$ An equally important finding in this "real-world" study was that only $68 \%$ of patients were treated with drugs recommended in the HMO's treatment guidelines based upon the Joint National Committee (JNC) recommendations. Others have found that members of HMOs and preferred provider organizations were more likely to use angiotensin-converting enzyme inhibitors (ACEIs) and calcium channel blockers (CCBs) for essential hypertension compared to fee-for-service health insurance and out-of-pocket purchasers who were more likely to use diuretics and beta-blockers, the latter consistent with JNC guidelines. ${ }^{43} \mathrm{~A}$ survey of 316 primary care physicians responding to a 26-item questionnaire in the year 2000 found that $41 \%$ reported little or no knowledge of JNC guidelines, at that time in its sixth iteration. ${ }^{44}$ This lack of familiarity was also evidenced in the use of ACEIs as the most common first-line drug of choice for hypertension, contrary to both JNC guidelines dating back to version III (1984) that advocated the use of diuretics and beta-blockers as first-line therapy. ${ }^{45}$

Examination of the relative value of ACEIs, CCBs and angiotensin receptor blockers (ARBs) in the treatment of hypertension must include the context of the results of the Antihypertensive and Lipid-lowering Treatment to Prevent Heart Attack Trial (ALLHAT), released in December 2002. The ALLHAT study results suggest that most of the more than 40 million Americans with hypertension ${ }^{46}$ could be treated more effectively, more safely, and more cheaply with low-cost diuretics such as chlorthalidone and hydrochlorothiazide at a 